\title{
Editor's preface: the science of intervertebral disc replacement
}

\author{
Hans-Joachim Wilke $\cdot$ Stephen J. Ferguson
}

Accepted: 23 April 2012/Published online: 8 May 2012

(C) Springer-Verlag 2012

Total disc arthroplasty (TDA) has evolved into an extremely exciting technology over the last decade. After the initial enthusiasm, some disillusion has also spread, because the long-term results are not as good and reliable as hoped, and it has become evident that the development of an artificial disc is a big challenge [1-3]. Nevertheless, the number of disc prosthesis products on the market has grown rapidly in the recent years. Until now, many proposed solutions are fairly simple, compared to a real intervertebral disc, which has a very complex structure [46]. Most of the current designs are based on an articulating ball-and-socket concept, reminiscent of the conventional implant systems that we already know from other joints. The various prosthesis designs attempt to control the movement directly by the geometry of the articulating surfaces, to mimic the physiological centre of rotation or to allow a fully unconstrained motion of the individual prosthesis components in devices with mobile cores. The current trend for disc implants is to design them in such a way that the three-dimensional physiological stiffness of the natural disc is reproduced. Therefore, the types of prostheses, which are currently available can be distinguished between implants with a fixed centre of rotation, incompressible prostheses with a mobile centre of rotation, and compressible prostheses with a mobile centre of rotation.

H.-J. Wilke ( $\square)$

Institute of Orthopaedic Research and Biomechanics,

University of Ulm, Helmholtzstrasse 14, 89081 Ulm, Germany

e-mail: hans-joachim.wilke@uni-ulm.de

\section{S. J. Ferguson}

Institute for Biomechanics, ETH Zurich,

Wolfgang-Pauli-Strasse 10, 8093 Zurich, Switzerland

e-mail: sferguson@ethz.ch
In order to advance the treatment with this still promising technology, we need to improve our understanding of the "Science of Intervertebral Disc Replacement". This supplement of the European Spine Journal publishes exclusively 15 original articles about in vitro studies, computational (FEM) simulations and wear analyses of artificial discs for both the lumbar and the cervical spine.

Some papers aim to elucidate the influence of not only conventional, but also so-called "second generation" prosthesis designs, on segmental biomechanics, to better guide the integration of such implants in the whole spine. The role of the posterior longitudinal ligament in lumbar total disc replacement is another topic of study, in order to provide advice on whether it should be resected or maintained.

Additional answers to similar questions about the indications and risks with multi-segmental approaches have been studied using mathematical methods or personalized dynamic biomechanical models driven by in vivo measurements.

The long-term survival of these implants remains an open question. Other papers describe wear-testing experiments with new materials, or determine the influence of testing frequency or the location of centre of rotation on the wear properties.

We hope this issue is of interest for both surgeons and scientists from other disciplines, who deal with the development and clinical application of artificial discs.

Conflict of interest None.

\section{References}

1. Mayer HM, Korge A (2002) Non-fusion technology in degenerative lumbar spinal disorders: facts, questions, challenges. Eur Spine J 11(Suppl 2):S85-S91 
2. Putzier M et al (2006) Charite total disc replacement—clinical and radiographical results after an average follow-up of 17 years. Eur Spine J 15(2):183-195

3. Schluessmann E et al (2009) SWISSspine: a nationwide registry for health technology assessment of lumbar disc prostheses. Eur Spine J 18(6):851-861

4. Galbusera $F$ et al (2008) Design concepts in lumbar total disc arthroplasty. Eur Spine J 17(12):1635-1650
5. Gunzburg R, Szpalski M (2002) Use of a novel beta-tricalcium phosphate-based bone void filler as a graft extender in spinal fusion surgeries. Orthopedics 25(5 Suppl):591-595

6. Szpalski M, Gunzburg R, Mayer M (2002) Spine arthroplasty: a historical review. Eur Spine J 11(Suppl 2):S65-S84 